\title{
Changes in mood states and salivary cortisol levels following two months of training in elite female water polo players
}

\author{
DONATELLA DI CORRADO ${ }^{1}$, TIZIANO AGOSTINI ${ }^{2}$, MARCO BONIFAZI $^{3}$ and VINCENZO PERCIAVALLE ${ }^{4}$ \\ ${ }^{1}$ Department of Sport Sciences, Kore University of Enna, Enna I-94100; ${ }^{2}$ Department of Life Sciences, University of Trieste, \\ Trieste I-34127; ${ }^{3}$ Department of Physiology, University of Siena, Siena I-43100; ${ }^{4}$ Department of Biomedical Sciences, \\ Section of Physiology, University of Catania, Catania I-95125, Italy
}

Received August 13, 2013; Accepted March 3, 2014

DOI: $10.3892 / \mathrm{mmr} .2014 .2115$

\begin{abstract}
Psychological and physiological markers have been used to maximize competitive sport preparation. The aim of the present study was to evaluate the effects of two months of training and competitions on mood states and salivary cortisol diurnal variation in 15 female elite water polo players. The results demonstrated that the $\beta$ coefficient values of the regression slope of cortisol values on sampling time was significantly flatter following the training period. The values of the area under the cortisol curve (AUC) 'with respect to ground' (AUCg) and those of the $\beta$ coefficients of the slope of the diurnal change in cortisol levels were highly negatively correlated. The slope correlated positively with the dysfunctional Profile of Mood States scale and negatively with the functional scale. AUCg correlated negatively with dysfunctional factors and positively with the vigor index. These findings support the hypothesis that subclinical hypocortisolism and a flattened diurnal rhythm of cortisol may play a role in determining dysfunctional mood states.
\end{abstract}

\section{Introduction}

Psychological and biological markers have been used to maximize competitive preparation (1-3). On the psychological side, tests able to measure specific psychological features and mood states have been used, while on the biological side, salivary cortisol analysis has been considered an efficient tool for monitoring the effects of training. Sport psychology researchers have relied almost exclusively upon the Profile of Mood States (POMS) scale (4) as the measure of mood when examining links with athletic performance. Morgan $(5,6)$ pioneered the use of the POMS in sport, and demonstrated that greater than

Correspondence to: Professor Vincenzo Perciavalle, Department of Biomedical Sciences, Section of Physiology, University of Catania, 6 Viale Andrea Doria, Catania I-95125, Italy

E-mail: perciava@unict.it

Key words: water polo, female athlete, cortisol, mood state, stress responses average vigor scores and lower than average scores for anger, tension, fatigue, depression and confusion characterized the mood profiles of athletes, particularly at the elite level, when compared with population norms. When an athlete undergoes intense physical training, the POMS usually reveals reduced vigor and increased tension, depression, anger, fatigue and confusion (7). Cortisol serves a wide range of physiological, behavioral and cognitive functions. Mineralocorticoid and glucocorticoid receptor distribution has been described in the primate amygdala, hippocampus, medial prefrontal and orbitofrontal cortical areas $(8,9)$, which are involved in perception, memory and the experience of emotional events. Evidence is accumulating that demonstrates that glucocorticoid dysfunction may contribute to mood disorders via extrahypothalamic region activation. Cortisol appears to exhibit cognitive and behavioral effects acting in a curvilinear, or 'inverted U-shaped,' manner in numerous physiological and cognitive systems, where moderate levels are optimal and where concentrations at extreme levels, either low or high, are represented by a distinct adverse cognitive or behavioral outcome (10). The cortisol concentration in the blood changes during the day, with the highest values in the early morning and a progressive decrease reaching the lowest values in the late evening. Free cortisol measured in the saliva has the same diurnal rhythm as plasma cortisol (11). Thus, the measurement of cortisol in the saliva is a reliable tool for investigating the activity of the hypothalamus-pituitary-adrenal (HPA) axis (12-14). Healthy cortisol rhythms are characterized by high morning and low evening levels, while dysregulated cortisol rhythms demonstrate flattened or aberrant daytime profiles. While there has been no precise delineation of the consequences of flattened cortisol rhythms in female athletes, normal females with flatter rhythms have been shown to deliver a poorer performance with regard to explicit memory tasks, and have lower perceived social support and an inclination to higher perceived stress (15). Acute intense exercise activates the HPA axis, and it has been reported that the adaptations to the HPA axis induced by endurance training cause an increase in basal plasma cortisol (16). However, intense physical training, by acting as a chronic repeated stress that dysregulates the stress response system, may lead also to reduced HPA axis reactivity (17). Limited information is available concerning the diurnal variations of salivary cortisol concentrations in 
athletes. A blunted diurnal rhythmicity of cortisol has been reported in males and females $(18,19)$. However, the reason for the reduced basal HPA activity in trained athletes remains unclear. Few studies have investigated the possible association between salivary cortisol levels and mood state during physical training. O'Connor et al (20) used the POMS questionnaire and resting salivary cortisol levels to assess the effect of progressive increases and decreases in training volume in female swimmers. Compared with the controls, the athletes reported alterations in depression, tension, anger, fatigue, vigor and global mood throughout the training season. At baseline and following increased training, the levels of salivary cortisol were significantly greater in the swimmers compared with the controls; this was significantly correlated with a depressed mood following increased training, however, not at baseline. Verde et al (21) observed the effects of overstress on POMS scores and cortisol levels by assessing highly trained distance runners who deliberately increased their training by $38 \%$ over a 3 week period. The study revealed that the POMS was the best single marker of disturbed function, indicating increased fatigue, while changes in cortisol were not statistically significant. Filaire et al (22) reported that in adolescent female tennis players, a lower overall cortisol output occurred following 16 weeks of training concomitantly with an increase in general stress and fatigue. It was hypothesized that chronic stress induced by training may lead to a dysregulated adaptation to exercise consisting of a subclinical form of hypocortisolism.

The main purpose of the present study was to monitor mood states and salivary cortisol diurnal rhythm in the first two months of training and competitions of the agonistic season in a group of female elite water polo players. The hypothesis was the existence of a difference in mood states and salivary cortisol levels between the first survey (two weeks prior to the beginning of the championship) and the second survey (within the first two months of the championship season). In particular, the present study predicted changes in diurnal rhythm and total output of cortisol concomitantly with an increase in dysfunctional mood states (tension-anxiety, depression-dejection, anger-hostility, fatigue-inertia and confusion-bewilderment) and a decrease in functional states (vigor-activity), in the $2^{\text {nd }}$ survey following the beginning of the championship. Furthermore, changes in mood states were hypothesized to be less pronounced in experienced players. In fact, in line with previous studies (23-25), experienced players were expected to cope better with stressful events.

\section{Materials and methods}

Participants. In total, 15 female water polo players, between the ages of 16-39 years old [mean \pm standard deviation (SD), 22.93 \pm 7.33 ] participated in the present study. The subjects were members of the 'Orizzonte Catania' team (winner of the national title for 10 consecutive years), who competed during the A1 Italian championship. Participants were considered healthy, injury-free and reported relatively consistent menstrual cycling of 27-32 days in duration. Their training schedule consisted of $14.5 \mathrm{~h}$ per week distributed in nine sessions, plus a competition game on the Saturday of each weekend (starting from October 16th). Four training sessions per week were devoted to technical and tactical drills, three sessions were devoted to swimming (mean amount, $\sim 8,000$ meters per week) and two sessions were performed in the gym for resistance exercises. The training work load remained almost unaltered during the period in which the water polo players were studied (October-December). The experienced players were considered to be the athletes with $>3$ years of experience in the A1 Italian championship. The group of experienced players consisted of nine players between the ages of 21 and 39 years old.

Mood measurement. Mood was measured using the POMS scale. The original version was developed in 1971 and consisted of 65 items. The present study used an abbreviated 30-item version of the POMS (4). The respondents must complete the POMS questionnaire by rating each item on a 5-point Likert scale with anchors ranging between 'Not at all' to 'Extremely'. Internal consistency is extremely high $(\mathrm{r}=0.90)$. The items are combined to form six separate subscales: Tension-anxiety, depression-dejection, anger-hostility, vigor-activity, fatigueinertia and confusion-bewilderment. The subscale scores may be combined to form an overall measure of affect that is labeled as total mood disturbance (TMD). The separate subscales are useful when researchers are interested in examining changes in specific moods. TMD is useful in studies containing a small number of participants or when researchers are interested in a single, global estimate of affective states. There are four response sets that can be employed with the POMS, and the 'today' set (i.e., 'How are you feeling today?') was used in the present study. The POMS questionnaire usually takes between 5-7 min to complete.

Salivary cortisol assay. Salivary cortisol measures were assessed using a radioimmunoassay cortisol test (Radim SpA, Pomezia, Rome, Italy). The method was founded on the competition between unlabeled cortisol and a fixed quantity of ${ }^{125}$ I-labeled cortisol for a limited number of binding sites on cortisol specific antibody. In this way, the amount of tracer bound by the antibody was inversely proportional to the concentration of unlabeled ligand. At the end of the incubation period, the liquid in the tubes was removed by aspiration and the radioactivity was measured using a $\gamma$ counter. The saliva was collected in sterile containers $(\sim 4 \mathrm{ml})$ over a timed collection period of $2 \mathrm{~min}$ and stored at $-70^{\circ} \mathrm{C}$ until use. Following thawing, the salivary sample was centrifuged for 5-10 min at $3,000 \mathrm{x}$ g. The clear colorless supernatant $(100 \mu \mathrm{l})$ was pipetted and dispensed in coated tubes to measure the salivary cortisol level.

Study protocol. To address the study hypotheses, the tests (salivary cortisol and POMS) were administered in two sessions at a time interval of two months (October 1-4, 2010, December 1-4, 2010). The first session was performed two weeks prior to the beginning of the A1 Italian championship, while the second session was executed a month and a half after the beginning of the championship (six games played). The saliva samples were collected three times per day (30 min after awakening and at 14:00 and 20:00 h; prior to breakfast, lunch and dinner, respectively) for three consecutive days. Each sample was executed by administering a tampon, which the athletes chewed for $\sim 1$ min. Every participant received 3 sterile containers that were previously marked with the 


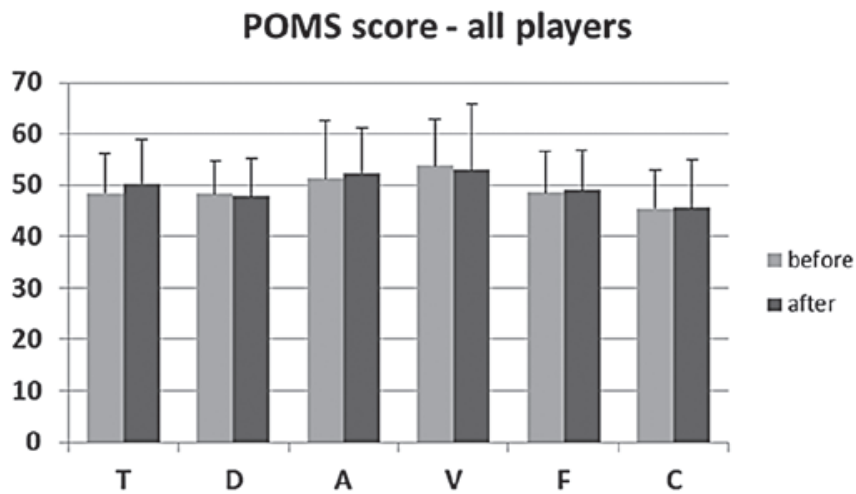

Figure 1. Mean \pm standard deviation (SD) values for the six subscales of POMS of all players prior to and following two months of training $(n=15)$. POMS, Profile of Mood States; T, tension-anxiety; D, depression-dejection; A, angerhostility; V, vigor-activity; F, fatigue-inertia; C, confusion-bewilderment.

\section{POMS score - experienced players}

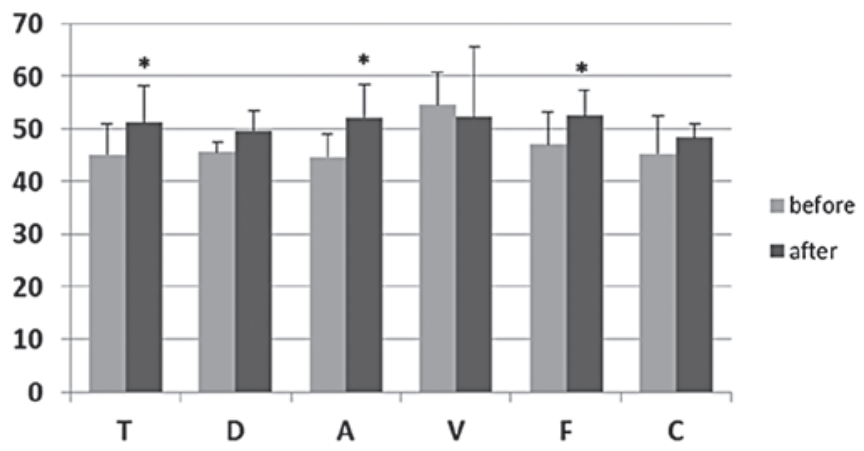

Figure 2. Mean \pm standard deviation (SD) values for the six subscales of POMS of experienced players prior to and following two months of training $(n=9)$. POMS, Profile of Mood States; T, tension-anxiety; D, depression-dejection; A, anger-hostility; V, vigor-activity; F, fatigue-inertia; C, confusion-bewilderment.

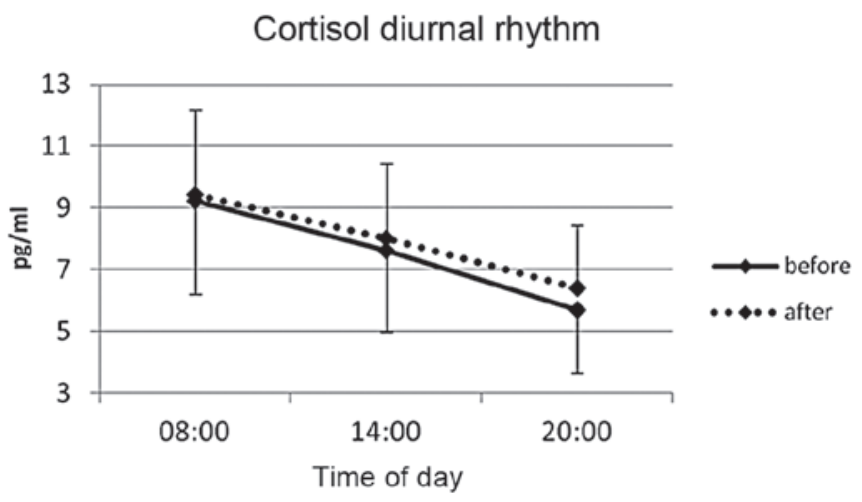

Figure 3. Mean \pm standard deviation (SD) concentration values of salivary free cortisol in water polo players at three time-points prior to and following two months of training.

identification code of the athlete, the date and the set time. The samples were frozen at $-70^{\circ} \mathrm{C}$ until use. Free cortisol in the saliva samples was determined using a radioimmunoassay, as aforementioned. Prior to assessment, the participants were instructed regarding the purpose of the study and were asked to follow the instructions for the collection of the saliva samples, including avoiding caffeine and acidic drinks and not brushing their teeth or eating or drinking anything $15 \mathrm{~min}$ prior to a sample collection. On day 4, following the initiation of the study, each athlete was asked to fill in the POMS questionnaire.

Statistical analysis. Data are presented as the mean \pm SD. The slope of the diurnal change in cortisol level was calculated to estimate how each player fit the normal (i.e., descending) profile. A linear regression of the three cortisol values on the sampling times was calculated, with data pooled for each player. Steeper slopes represented smaller $\beta$ coefficient values for the slope of the regression, indicating that the decrease in cortisol was more rapid. Flatter slopes (larger $\beta$ coefficient values) indicated slower decreases or abnormally timed peaks. It has been reported that this pattern may be due to a loss of resiliency of the HPA axis (26). The average area under the curve (AUC) was also calculated by trapezoidal estimation using cortisol values. Using the formulae outlined by Pruessner et al (27), the AUC 'with respect to ground' (AUCg) was calculated, which, in accordance with the interpretation provided by the authors, provides information regarding the total hormonal output and thus the basal activity of the HPA axis of the players. Statistical analysis was performed with an SPSS package (version 16.0; SPSS, Inc., Chicago, IL, USA) and all the statistical requirements for the used tests were fulfilled. The data collected prior to and following the training period were evaluated with the t-test for paired data and the Pearson's correlation test. $\mathrm{P}<0.05$ was considered to indicate a statistically significant difference for all comparisons.

Ethics. The procedures were conducted in accordance with the Declaration of Helsinki and were performed with ethics approval by the University of Catania (Catania, Italy). Each participant was provided with a full explanation of the protocols, and written informed consent was obtained prior to commencement of the study.

\section{Results}

When considering the data from all players, the scores of the six separate subscales of POMS did not change following the assessed period (Fig. 1). However, when considering only the subgroup of experienced players, the score of tension-anxiety, anger-hostility and fatigue-inertia increased significantly (Fig. 2). Fig. 3 demonstrates the diurnal rhythm of grand mean salivary free cortisol observed at each sampling time prior to and following the training period. No changes were observed in the AUCg values (mean $\pm \mathrm{SD}$ ) following the assessed period in the whole group of players (before, 15.1 \pm 5.1 ; and after, $15.9 \pm 4.8 ; \mathrm{P}=0.41)$ and in the subgroup of experienced players (before, 15.9 \pm 5.3 ; and after, 15.4 $\pm 5.4 ; \mathrm{P}=0.64$ ). By contrast, the slope of the diurnal change in cortisol levels (mean $\beta$ coefficient values \pm SD) decreased significantly in the whole group of players (before, $-0.253 \pm 0.078$ vs. $-0.217 \pm 0.052$; $\mathrm{P}<0.05)$, indicating that the decrease in cortisol was less rapid in the second survey. The slope was flatter also when considering the subgroup of experienced players (before, $-0.265 \pm 0.086$ vs. $-0.208 \pm 0.059 ; \mathrm{P}<0.01)$. In the whole group of players, the values of $\mathrm{AUCg}$ and those of the $\beta$ coefficients of 
Table I. Correlation coefficients of the linear regressions among the individual scores of the six subscales of POMS and the values of AUCg and $\beta$ coefficient of the slope prior to and following two months of training.

\begin{tabular}{lccccc}
\hline & \multicolumn{2}{c}{ AUCg } & & \multicolumn{2}{c}{ Slope $\beta$ values } \\
\cline { 2 - 3 } \cline { 6 - 7 } Subscales & Before & After & & Before & After \\
\hline T & $-0.82^{\mathrm{a}}$ & $-0.82^{\mathrm{a}}$ & & $0.79^{\mathrm{a}}$ & $0.90^{\mathrm{a}}$ \\
$\mathrm{D}$ & $-0.54^{\mathrm{b}}$ & $-0.55^{\mathrm{b}}$ & & $0.53^{\mathrm{b}}$ & $0.58^{\mathrm{b}}$ \\
$\mathrm{A}$ & -0.51 & $-0.38^{\mathrm{n}}$ & & 0.46 & $0.57^{\mathrm{b}}$ \\
$\mathrm{V}$ & $0.68^{\mathrm{a}}$ & $0.74^{\mathrm{a}}$ & & $-0.55^{\mathrm{b}}$ & $-0.65^{\mathrm{a}}$ \\
$\mathrm{F}$ & $-0.67^{\mathrm{a}}$ & $-0.64^{\mathrm{b}}$ & & $0.56^{\mathrm{b}}$ & $0.61^{\mathrm{b}}$ \\
$\mathrm{C}$ & $-0.52^{\mathrm{b}}$ & $-0.64^{\mathrm{b}}$ & & 0.36 & 0.43 \\
\hline
\end{tabular}

${ }^{\mathrm{a}} \mathrm{P}<0.01 ;{ }^{\mathrm{b}} \mathrm{P}<0.05(\mathrm{n}=15)$. POMS, Profile of Mood States; AUCg, area under the cortisol curve 'with respect to ground'; T, tension-anxiety; $\mathrm{D}$, depression-dejection; A, anger-hostility; V, vigor-activity; $\mathrm{F}$, fatigue-inertia; $\mathrm{C}$, confusion-bewilderment

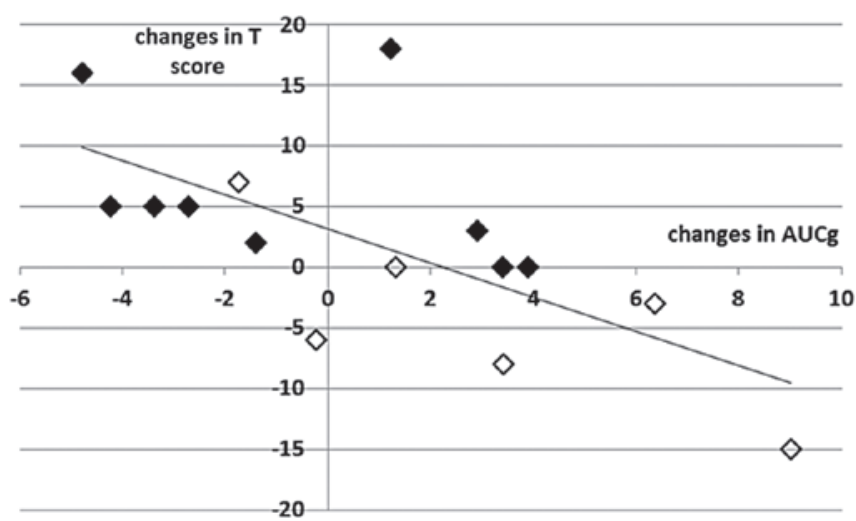

Figure 4. Linear regression between changes in tension-anxiety scores and changes in AUCg values following two months of training. AUCg, area under the cortisol curve 'with respect to ground'.

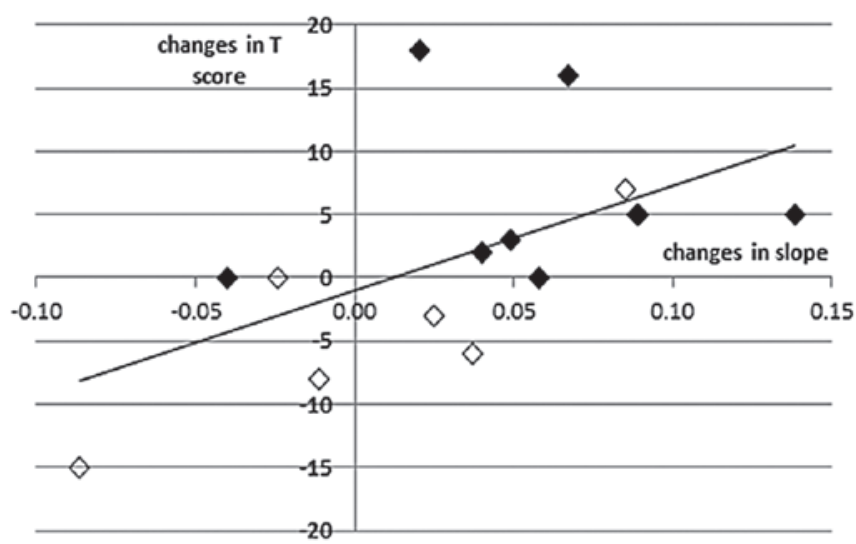

Figure 5. Linear regression between changes in tension-anxiety scores and changes in $\beta$ coefficient values of the slope following two months of training.

the slope of the diurnal change in cortisol levels were highly negatively correlated between them $(r=-0.92 ; \mathrm{P}<0.001)$, i.e., a higher cortisol output and steeper slope of the diurnal rhythm.
Table I demonstrates the correlation coefficients of the linear regression among the scores of the six separate subscales of POMS and the values of AUCg and $\beta$ coefficient values of the slope in the whole group. Significant negative correlations were identified among scores of dysfunctional scales of POMS (with the exception of anger-hostility) and AUCg, while vigor-activity was positively correlated with this. By contrast, positive correlations were identified among scores of dysfunctional scales of POMS and $\beta$ coefficient values of the slope, while vigoractivity was negatively correlated with the same parameter. The changes in the score of the tension-anxiety subscale were negatively $(\mathrm{r}=-0.67 ; \mathrm{P}<0.01)$ correlated with changes in $\mathrm{AUCg}$ (Fig. 4) and positively $(\mathrm{r}=0.57 ; \mathrm{P}<0.05)$ correlated with the changes in the $\beta$ coefficient values of the slope of the diurnal variation in cortisol levels (Fig. 5) following the assessed period in the whole group. A rise in cortisol total output corresponds to a decrease in the tension-anxiety score, while a flatter slope corresponds to an increase in the same score. Similar correlations were also identified among the changes in the score of the fatigue-inertia subscale and changes in AUCg and $\beta$ coefficient values ( $\mathrm{r}=-0.55$ and $\mathrm{r}=0.52$, respectively; $\mathrm{P}<0.05$ for both).

\section{Discussion}

The main purpose of the present study was to monitor mood states and salivary cortisol in the first two months of training and competitions in a group of female elite water polo players. First of all, considering the slope of the diurnal rhythm in cortisol level, a significant decrease was revealed following the considered period in all players. Analyzing the correlations between the scores of the six separate subscales of POMS and the values of AUCg and $\beta$ coefficient values of the slope in the whole group, significant negative correlations were identified among scores of dysfunctional scales of POMS (with the exception of anger-hostility) and AUCg, while vigor-activity was positively correlated with this. By contrast, positive correlations were identified among the scores of dysfunctional scales of POMS and the $\beta$ coefficient values of the slope, while vigor-activity was negatively correlated with the same parameter. These results support our hypothesis that POMS scales are good individual indicators of diurnal change in cortisol levels expressed by the AUCg and the slope. In the present study, the total cortisol output, as expressed by AUCg values, did not change following two months of training. This finding differs from that revealed by Filaire et al (22), which observed a lower overall AUCg in adolescent female tennis players following 16 weeks of training. This discrepancy may be explained by the lower duration of the training period in the present study. However, following two months of training, the observed decrease in the slope of the diurnal rhythm of cortisol indicates a loss of resiliency of the HPA axis. Similarly, Georgopoulos et al (18) demonstrated an abolished diurnal rhythm of salivary cortisol in elite male and female artistic gymnasts, while a blunted diurnal rhythmicity of cortisol in young male dancers was also reported by Strahler et al (19). The decrease of resiliency of the HPA axis is considered to be associated with protracted exposure to stress, perhaps due to hippocampal impairment of feedback inhibition (28). The loss of feedback function may be mediated by decreased glucocorticoid receptor function $(26,29)$ which has been recently demonstrated in highly trained swimmers (30). 
In the present study, the players demonstrating the greatest decrease in the slope of diurnal rhythm of cortisol also had the greatest reduction in total cortisol output. Thus, at least in certain players, a dysregulated adaptation to training, evolving in a subclinical form of hypocortisolism, occurred. In fact, hypocortisolism does not appear to be an exclusive correlate of stress-related pathology, but has also been reported in healthy subjects subjected to living with ongoing stress. A previous study in in humans indicated reduced adrenocortical activity or reactivity in states of chronic stress (31). Such modifications may be due to various mechanisms, including increased negative feedback inhibition of the HPA axis, decreased responsiveness of cortisol secretion and/or reduced biosynthesis or depletion at several levels of the HPA axis. Besides these basic mechanisms, superimposed factors, including the nature of the stressor, coping styles and dispositions, may determine the manifestation of hypocortisolism (31). It should be highlighted that moderately elevated cortisol is considered to be advantageous for increased arousal and energy expenditure in the organization of behavior. Cortisol is also important for efficient cognitive appraisal of events and is known to aid in the neural processing that occurs in the brain regions that are responsible for processing information with regard to emotional events (10). The present study demonstrated that hypocortisolism, which is characterized by a flat diurnal cortisol slope, was associated with higher values in POMS dysfunctional scales, thus supporting the importance of cortisol in sustaining and facilitating cognitive functions. However, no difference was identified in the scores of the six separate subscales of POMS when considering all players together. When considering only the experienced players, the score of tension-anxiety, anger-hostility and fatigue-inertia increased significantly following two months of training. These findings are not in line with previous studies that indicated that experts cope better with stressful events (23-25). However, the present results may be explained by the fact that the team had won the national title for 10 consecutive years. In consequence of this, the experienced athletes may have felt more responsible for the final result of the team and therefore they may be more exposed to higher stress levels leading to a possible dysregulation of the HPA axis. Furthermore, it must be noted that, 10 days prior to the second survey, the team lost one game. In this respect, it has been reported that expert athletes compared with novices, through years of practice and coping with pressure experiences, develop coping strategies that enable them to remain in their preferable emotional-attentional state. Once this equilibrium state is interrupted, a detrimental effect on the emotional states of athletes may occur (3). However, following the second survey, the team won seven games consecutively, indicating that the dysregulated state of the HPA axis concomitant with high values in POMS dysfunctional scales, as observed in certain players, were possibly of a transitional nature and, however, not able to adversely affect the performance.

There are a number of limitations to the present study. Firstly, it is difficult to generalize the present findings from elite female players to athletes of other genders, sports or fitness levels. Although the literature findings are not uniformly consistent, evidence from several studies indicates that there is a gender difference with respect to HPA axis function in humans. Decreased basal cortisol concentrations and enhanced suppression of cortisol by dexamethasone have been measured in females in the follicular phase of the menstrual cycle relative to females in the luteal phase or compared with males (31). Observed gender differences in HPA axis stress responses may be due to sexual dimorphism in brain function and circulating sex steroids (32). Secondly, a small sample was studied; however, it must be noted that it is difficult to recruit a homogeneous sample of high-level athletes. Thirdly, when investigating elite sport activities, training effects may occur from day to day. Despite these limitations, the results of the present study offer support for the hypothesis that hypocortisolism and a flattened diurnal rhythm plays a significant role in determining dysfunctional mood states. In this respect, the POMS is confirmed to be a potent, reliable and valid measure of mood in physical activity settings. One primary advantage of the POMS is that it appears to be useful in detecting mood fluctuations associated with exercise. Another advantage of the POMS is that the six subscales appear to be measuring mood subcomponents, which are differentially responsive to diverse characteristics of exercise settings (33). However, when approaching psychological evaluation in elite athletes, it must be considered that personality factors and cognitive appraisal processes are not only important in determining what is stressful, but also in modulating the extent and habituation of the HPA axis response to stress (34). Thus, further investigation is required to determine specific personality constructs that may distinguish which individuals are more susceptible to psychological stress and mood disturbances (35).

\section{Acknowledgements}

The authors would like to thank the management of the Water Polo Club Orizzonte Catania for logistical support and the collaboration and patience of their players and coaches that contributed to this study.

\section{References}

1. Hellhammer DH and Hellhammer J (eds): Neurobehavioral medicine and stress-related disorders. In: Stress: The Mind-Body Connection. Vol 174. Karger Publishers, Basel, pp1-10, 2008.

2. Kellmann M (ed): Enhancing recovery: preventing underperformance in athletes. 1st edition. Human Kinetics Publishers, Champaign, IL, pp340, 2002.

3. Tenenbaum G, Edmonds WA and Eccles DW: Emotions, coping strategies, and performance: a conceptual framework for defining affect-related performance zones. Mil Psychol 20: S11-S37, 2008.

4. McNair DM, Lorr M and Droppleman LF (eds): Profile of Mood States Manual. Education and Industrial Testing Service, San Diego, CA, pp40, 1992.

5. Morgan WP: Selected psychological considerations in sport. Res Q 45: 374-390, 1974.

6. Morgan WP and Pollock ML: Psychological characterization of the elite distance runner. Ann NY Acad Sci 301: 383-403, 1977.

7. Koutedakis Y, Budgett R and Faulmann L: Rest in underperforming elite competitors. Br J Sports Med 24: 248-252, 1990.

8. Sánchez MM, Young LJ, Plotsky PM and Insel TR: Distribution of corticosteroid receptors in the rhesus brain: relative absence of glucocorticoid receptors in the hippocampal formation. J Neurosci 20: 4657-4668, 2000

9. Seckl JR, Dickson KL, Yates C and Fink G: Distribution of glucocorticoid and mineralocorticoid receptor messenger RNA expression in human postmortem hippocampus. Brain Res 561: 332-337, 1991.

10. Erickson K, Drevets W and Schulkin J (2003). Glucocorticoid regulation of diverse cognitive functions in normal and pathological emotional states. Neurosci Biobehav Rev 27: 233-246, 2003. 
11. Stone AA, Schwartz JE, Smyth J, Kirschbaum C, Cohen S, Hellhammer D and Grossman S: Individual differences in the diurnal cycle of salivary free cortisol: a replication of flattened cycles for some individuals. Psychoneuroendocrinology 26: 295-306, 2001.

12. Hellhammer DH, Wüst S and Kudielka BM: Salivary cortisol as a biomarker in stress research. Psychoneuroendocrinology, 34: 163-171, 2009.

13. Levine A, Zagoory-Sharon O, Feldman R, Lewis JG and Weller A: Measuring cortisol in human psychobiological studies. Physiol Behav 90: 43-53, 2007.

14. Vining RF, McGinley RA, Maksvytis JJ and Ho KY: Salivary cortisol: a better measure of adrenal cortical function than serum cortisol. Ann Clin Biochem: 20: 329-335, 1983.

15. Abercrombie HC, Giese-Davis J, Sephton S, Epel ES, Turner-Cobb JM and Spiegel D: Flattened cortisol rhythms in metastatic breast cancer patients. Psychoneuroendocrinology 29: 1082-1092, 2004.

16. Duclos M, Corcuff JB, Arsac L, Moreau-Gaudry F, Rashedi M, Roger P, Tabarin A and Manier G: Corticotroph axis sensitivity after exercise in endurance-trained athletes. Clin Endocrinol (Oxf) 48: 493-501, 1998

17. Urhausen A, Gabriel HH and Kindermann W: Impaired pituitary hormonal response to exhaustive exercise in overtrained endurance athletes. Med Sci Sports Exerc 30: 407-414, 1998.

18. Georgopoulos NA, Rottstein L, Tsekouras A, et al: Abolished circadian rhythm of salivary cortisol in elite artistic gymnasts. Steroids 76: 353-357, 2011.

19. Strahler J, Berndt C, Kirschbaum C and Rohleder N: Aging diurnal rhythms and chronic stress: Distinct alteration of diurnal rhythmicity of salivary alpha-amylase and cortisol. Biol Psychol 84 248-256, 2010.

20. O'Connor PJ, Morgan WP, Raglin JS, Barksdale CM and Kalin NH: Mood state and salivary cortisol levels following overtraining in female swimmers. Psychoneuroendocrinology 14: 303-310, 1989.

21. Verde T, Thomas S and Shephard RJ: Potential markers of heavy training in highly trained distance runners. Br J Sports Med 26 167-175, 1992.

22. Filaire E, Ferreira JP, Oliveira M and Massart A: Diurnal patterns of salivary alpha-amylase and cortisol secretion in female adolescent tennis players after 16 weeks of training. Psychoneuroendocrinology 38: 1122-1132, 2013

23. Cleary TJ and Zimmerman BJ: Self-regulation differences during athletic practice by experts, non-experts, and novices. J Appl Sport Psychol 13: 185-206, 2001.
24. Kitsantas A and Zimmerman BJ: Comparing self-regulatory processes among novice, non-expert, and expert volleyball players: A microanalytic study. J Appl Sport Psychol 14: 91-105, 2002

25. Tamminen KA and Holt NL: Female adolescent athletes' coping: a season-long investigation. J Sports Sci 28: 101-114, 2010.

26. Bonifazi M, Suman AL, Cambiaggi C, et al: Changes in salivary cortisol and corticosteroid receptor-alpha mRNA expression following a 3 -week multidisciplinary treatment program in patients with fibromyalgia. Psychoneuroendocrinology 31: 1076-1086, 2006.

27. Pruessner M, Hellhammer DH, Pruessner JC and Lupien SJ: Self-reported depressive symptoms and stress levels in healthy young men: associations with the cortisol response to awakening. Psychosom Med 65: 92-99, 2003.

28. Crofford LJ, Young EA, Engleberg NC, et al: Basal circadian and pulsatile ACTH and cortisol secretion in patients with fibromyalgia and/or chronic fatigue syndrome. Brain Behav Immun 18: 314-325, 2004.

29. Otte C, Yassouridis A, Jahn H, et al: Mineralocorticoid receptormediated inhibition of the hypothalamic-pituitary-adrenal axis in aged humans. J Gerontol A Biol Sci Med Sci 58: B900-B905, 2003.

30. Bonifazi M, Mencarelli M, Fedele V, et al: Glucocorticoid receptor mRNA expression in peripheral blood mononuclear cells in high trained compared to low trained athletes and untrained subjects. J Endocrinol Invest 32: 816-820, 2009.

31. Heim C, Ehlert U and Hellhammer DH: The potential role of hypocortisolism in the pathophysiology of stress-related bodily disorders. Psychoneuroendocrinology 25: 1-35, 2000.

32. Aloisi AM and Bonifazi M: Sex hormones, central nervous system and pain. Horm Behav 50: 1-7, 2006.

33. Berger BG and Motl RW: Exercise and mood: a selective review and synthesis of research employing the profile of mood states. J Appl Sport Psychol 1: 69-92, 2000.

34. Gaab J, Blattler N, Menzi T, et al: Randomized controlled evaluation of the effects of cognitive-behavioral stress management on cortisol responses to acute stress in healthy subjects. Psychoneuroendocrinology 28: 767-779, 2003.

35. Smyth J, Ockenfels MC, Porter L, et al: Stressors and mood measured on a momentary basis are associated with salivary cortisol secretion. Psychoneuroendocrinology 23: 353-370, 1998. 